\title{
Toward In Vivo Transdermal pH Sensing with a Validated Microneedle Membrane Electrode
}

\author{
Juan José García-Guzmán, Clara Pérez-Ràfols, María Cuartero, and Gastón A. Crespo* \\ Cite This: https://dx.doi.org/10.1021/acssensors.0c02397 \\ Read Online
}

ACCESS

Llll Metrics \& More

Article Recommendations

Supporting Information

ABSTRACT: We present herein the most complete characterization of microneedle $(\mathrm{MN})$ potentiometric sensors for $\mathrm{pH}$ transdermal measurements for the time being. Initial in vitro assessment demonstrated suitable analytical performances (e.g., Nernstian slope, linear range of response from 8.5 to 5.0, and fast response time) in both buffer media and artificial interstitial fluid (ISF). Excellent repeatability and reproducibility together with adequate selectivity and resiliency facilitate the appropriateness of the new $\mathrm{pH} \mathrm{MN}$ sensor for transdermal ISF analysis in healthcare. The ability to resist skin insertions was evaluated in several ex vivo setups using three different animal skins (i.e., chicken, pork, and rat). The developed $\mathrm{pH} M N$ sensor was able to withstand from 5 to 10 repetitive insertions in all the skins considered with a minimal change in the calibration graph ( $<3 \%$ variation in both slope and intercept after the insertions). Ex vivo $\mathrm{pH}$ measurements were validated by determining the $\mathrm{pH}$ with the $\mathrm{MN}$ sensor and a commercial $\mathrm{pH}$ electrode in chicken skin portions previously conditioned at several $\mathrm{pH}$ values, obtaining excellent results with an accuracy of $<1 \%$ and a precision of $<2 \%$ in all cases. Finally, $\mathrm{pH} \mathrm{MN}$ sensors were applied for the very first time to transdermal measurements in rats together with two innovative validation procedures: (i) measuring subcutaneous $\mathrm{pH}$ directly with a commercial $\mathrm{pH}$ microelectrode and (ii) collecting ISF using hollow MNs and then the $\mathrm{pH}$ measurement of the sample with the $\mathrm{pH}$ microelectrode. The $\mathrm{pH}$ values obtained with $\mathrm{pH} \mathrm{MN}$ sensors were statistically more similar to subcutaneous measurements, as inferred by a paired sample $t$-test at $95 \%$ of confidence level. Conveniently, the validation approaches could be translated to other analytes that are transdermally measured with MN sensors.

KEYWORDS: ion-selective electrodes, $p H$ sensing, in vivo monitoring, microneedle transdermal sensing, validation

$\mathrm{M}$ icroneedle $(\mathrm{MN})$ sensors are attracting increasing attention linked to the need for new wearable diagnostic tools within the healthcare system. ${ }^{1,2}$ Indeed, the intrinsic ability of $\mathrm{MN}$ sensors to perform minimally invasive on-body and realtime measurements is crucial to accelerate the provision of meaningful observations in view of the next generation of personalized and preventive medicine. Unlike other formats of wearable chemical sensors that target "excretable" biological fluids such as sweat, tears, and saliva (e.g., sweat bands and patches, smart watches, glasses, and contact lenses), ${ }^{3-6} \mathrm{MN}$ sensors are primarily conceived for transdermal monitoring of interstitial fluid (ISF). Advantageously, the ISF composition has claimed to be very similar to blood, as a consequence of the existing equilibrium between ISF and plasma carried out by several small molecules (e.g., albumin, $\mathrm{CO}_{2}$, and phosphates). ${ }^{2}$ This similarity in composition is appealing for clinical purposes in order to substitute current (and inconvenient for the patient) blood analysis while providing the same physiological outcomes. In addition, biofouling in ISF is known to be lower than in blood due to lower concentration of proteins and other large molecules. $^{2,7,8}$

The analysis of ISF entails several challenges rarely addressed in the literature such as resistance to skin penetration and strict analytical validation (mostly absent) of the obtained analyte levels. ${ }^{2}$ It is essential to unequivocally demonstrate that the chemical sensing element embedded in the MN is not altered during skin insertion prior to claim any suitability for further in vivo applications; otherwise, the developed device may create unreal expectations. Special attention should be paid when the $\mathrm{MN}$ is externally modified with the sensing element because this is totally exposed to the skin penetration process. Therefore, external modification presents an increasing probability of detachment/alteration together with incremental cytotoxicity risk in the monitored subject compared to internal modification of the MN. ${ }^{2}$

Concerning the analytical validation of transdermal measurements through MNs, the main problem lies in the difficulty of extracting enough volume of ISF to be measured using a

Received: November 17, 2020

Accepted: February 1, 2021 
reference analytical technique. Several strategies have been reported for this purpose including hollow MNs, effusion, dialysis, sonication, and reverse iontophoresis. ${ }^{2,9}$ The latter has been demonstrated for the successful analysis of glucose but is not valid for all analytes because the iontophoresis process per se modifies the composition of the collected ISF. ${ }^{9}$ By contrast, extraction with hollow MNs preserves ISF composition as far as pertinent precautions are fulfilled to avoid sample evaporation and/or external contamination, which is a common issue whatever the collection method. The main limitation of ISF collection with MNs is that the volume is very low, usually less than $10 \mu \mathrm{L} .{ }^{10,11}$ Thus, in view of this problem, most in vivo studies using $\mathrm{MN}$ sensors attempt a validation by inducing changes in analyte concentration ${ }^{12,13}$ or comparing with concentration in blood, which has been widely applied in the case of $\mathrm{MNs}$ for glucose detection. ${ }^{14,15}$ Evidently, these strategies cannot be considered as pure analytical validation methods because they are either qualitative or do not consider the same fluid.

A search into the literature reveals that in vivo implementation of $\mathrm{MN}$ chemical sensors primarily targets glucose, with $\mathrm{pH}$ being the second analyte most widely detected. ${ }^{2}$ While the interest of measuring glucose to manage diabetes is undeniable, it has been proved that acid/base disorders are closely related to many diseases such as renal failure, ischemia, multiple sclerosis, and psychiatric disorders. Therefore, monitoring in vivo dynamic changes in $\mathrm{pH}$ has been claimed to be highly relevant from a clinical perspective. ${ }^{16}$ While a great number of $\mathrm{pH} \mathrm{MN}$ sensors can be found in the literature, with electrochemical readout but also others, ${ }^{2,17,18}$ to the best of our knowledge, only few works reported on real applications involving the in vivo determination of $\mathrm{pH}$ and transdermal measurements in ISF totally lack.

In this context, Mani et al. analyzed $\mathrm{pH}$ in mouse cerebrospinal fluid (CSF) and bladders using tungsten-modified MNs $(\mathrm{W} / \mathrm{ZnO})^{16}$ while Zuliani et al. developed an iridium oxide ( $\mathrm{IrOx}$ ) $\mathrm{MN}$ sensor for mapping $\mathrm{pH}$ distributions in rat heart. ${ }^{19}$ Zhou et al. developed a $\mathrm{pH}$ sensor based on molybdenum disulfide $\left(\mathrm{MoS}_{2}\right)$ nanosheets and polyaniline (PANI)-functionalized acupuncture needles for real-time monitoring of $\mathrm{pH}$ changes in rat brain. ${ }^{20}$ However, these papers did not cover the determination of $\mathrm{pH}$ in ISF and lack the evaluation of a possible detachment of the sensing element with the transdermal use of the $\mathrm{MN}$ and how this may affect the monitored individual. In this sense, it is important to mention that all the tungsten compounds are regarded as highly toxic compounds, ${ }^{21}$ whereas very little information is available for IrOx and the PANI- $\mathrm{MoS}_{2}$ tandem despite careful manipulation of these compounds being generally advised. ${ }^{22,23}$ In addition, the high-cost and high-temperature processing associated to metal oxide-based $\mathrm{pH}$ sensors is known to limit the fabrication of disposable $\mathrm{MN}$ sensors. ${ }^{24}$

In analogy to solid-contact electrochemical sensors already well-entrenched for $\mathrm{pH}$ detection in diverse samples, potentiometric ion-selective electrodes (ISEs) based on plasticized polymeric membranes should be good candidates toward effective transdermal monitoring of $\mathrm{pH}$ in ISF, beyond the use of inorganic coverings that are $\mathrm{pH}$-responsive (i.e., $\mathrm{W} / \mathrm{ZnO}$, IrOx, and PANI- $\left.\mathrm{MoS}_{2}\right){ }^{24,25}$ However, to the best of our knowledge, this approach has not been translated to the $\mathrm{MN}$ configuration yet. Importantly, our group has recently demonstrated the huge potential of the ISE technology toward potassium detection in ISF. ${ }^{26}$ Commercially available stainlesssteel solid MNs were externally modified, first with the solid ion- to-electron transducer and then with the ion-selective membrane (ISM), following a procedure adapted from the traditional all-solid-state ISE fabrication. ${ }^{27}$ The proposed MN technology demonstrated excellent resiliency to skin penetration and the absence of biofouling (in the time scale of hours). ${ }^{26}$ In addition, cytotoxicity studies using human dermal fibroblasts revealed a connection with the leaching of the ionophore from the ISM to the cell culture with any found toxic effect. ${ }^{28}$ Thus, while a toxic impact was detected in the 24-36 h experimental time frame for potassium and ammonium ISMs (based on valinomycin and nonactin, respectively), no effect appeared for other ionophores, including hydrogen ionophore I (typically used in $\mathrm{pH}$ sensors). ${ }^{28}$ In principle, these results pointed out the suitability of $\mathrm{pH}$-responsive ISMs to be implemented into $\mathrm{MN}$ technology for transdermal ISF analysis. This approach is expected to exceed the sensor biocompatibility compared to already existing $\mathrm{pH}$ MN sensors commented above. ${ }^{2,16,19,20}$

We present herein the very first $\mathrm{MN}$ sensor applied to the transdermal detection of $\mathrm{pH}$ in ISF in rats. It herein shows a deep characterization and pioneering validation protocol for $\mathrm{pH} M N$ sensors, which is schematized in Figure 1 and could pave the way

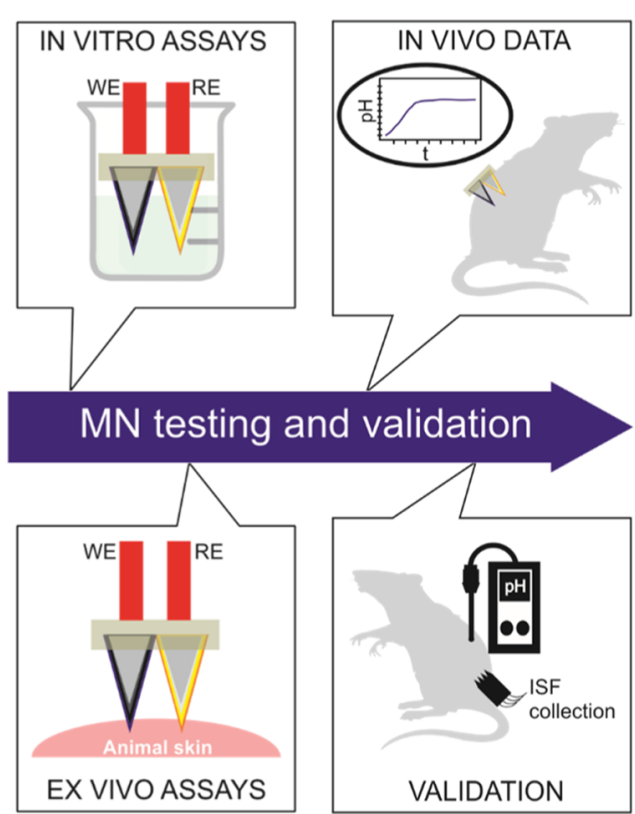

Figure 1. Scheme for the protocol used in the present paper for the characterization and validation of the $\mathrm{pH} \mathrm{MN}$ patch.

as a general strategy for any $\mathrm{MN}$ chemical sensor validation. In addition, the present work innovates toward proper inquiry of $\mathrm{MN}$ sensing technology through the in vitro, ex vivo, and in vivo journey aiming at reaching on-body measurements. Initial in vitro assessment is performed to evaluate the analytical performances of the developed $\mathrm{pH} \mathrm{MN}$ sensors in controlled buffer media and artificial ISF (AISF). Subsequently, the ability of the $\mathrm{MN}$ sensor to perform transdermal measurements is evaluated using several animal skins and following two protocols. The first one compares pre- and postinsertion calibrations in the skin to ensure that the sensing element is not damaged. The second protocol aims at testing the accuracy of transdermal $\mathrm{pH}$ measurements in skin pieces conditioned at different $\mathrm{pHs}$, that is, with known analyte concentration. Finally, $\mathrm{pH} \mathrm{MN}$ sensors are tested in rats together with two different validation approaches based on subcutaneous measurements 

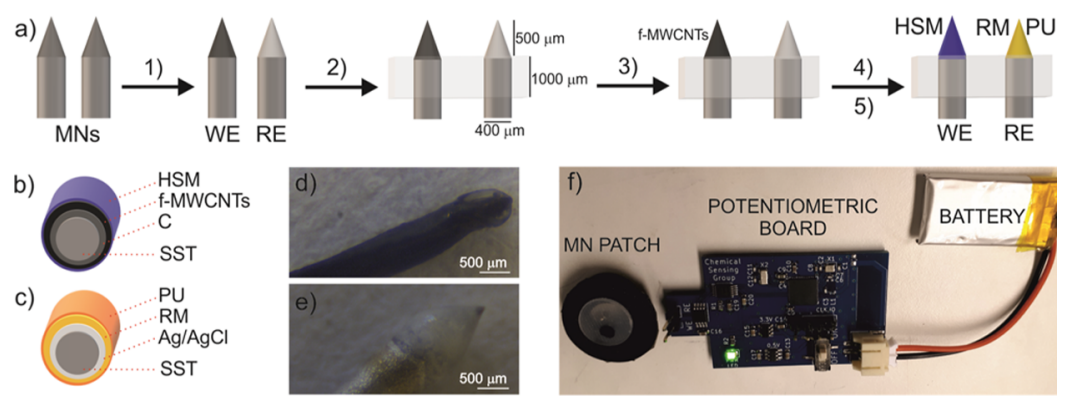

Figure 2. (a) Manufacturing procedure for the $\mathrm{MN}$ patch containing both the $\mathrm{pH}$-selective electrode and reference electrode: (1) stainless-steel solid $\mathrm{MNs}$ are modified with carbon and $\mathrm{Ag} / \mathrm{AgCl}$ ink coatings to further produce the working $(\mathrm{WE})$ and reference electrode (RE), respectively; (2) modified MNs are assembled into the silicon substrate; (3) deposition of f-MWCNTs on top of the carbon ink in the WE, (4) deposition of the pHselective membrane (HSM) in the WE; and (5) deposition of the RM and PU external layer in the RE electrode. (b) Cross-sectional image of the layers' arrangement in the WE, namely, stainless-steel core, carbon ink, f-MWCNTs, and HSM. (c) Cross-sectional image of the layers' arrangement in the RE: stainless-steel core, $\mathrm{Ag} / \mathrm{AgCl}$ ink, RM, and PU. (d) Image obtained by optical microscopy for the WE. (e) Image obtained by optical microscopy for the RE. (f) Arrangement of the entire device composed of the MN patch connected to the hand-made wireless potentiometric board.
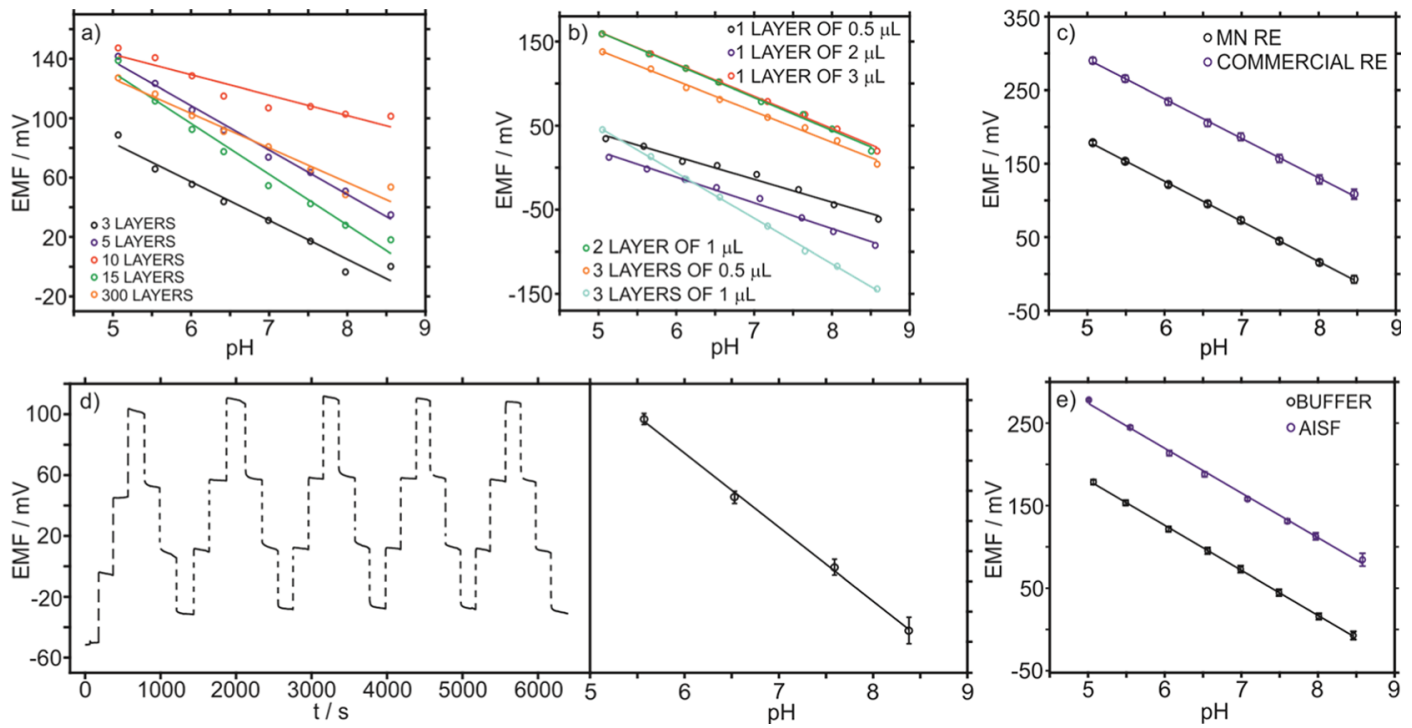

Figure 3. Calibration graphs obtained with two different deposition methods for the HSM incorporation: (a) dip-coating and (b) drop-casting. (c) Calibration curves obtained with the $\mathrm{pH} \mathrm{MN}$ sensor against a commercial $\mathrm{Ag} / \mathrm{AgCl} \mathrm{RE}$ and the developed $\mathrm{MN} \mathrm{RE}$. (d) Dynamic response and average calibration graph for the carry-over test (ten consecutive calibrations by alternatively increasing and decreasing the $\mathrm{pH}$ in buffer solutions). (e) Calibration curves obtained with the MN patch in two different backgrounds: buffer solutions and AISF at increasing $\mathrm{pH}$ values.

with a commercially available microelectrode and ISF extraction with bare hollow MNs. Advantageously, the procedure to fabricate and validate the $\mathrm{pH} \mathrm{MN}$ sensors can be tailored for other ions and molecules, therefore prospecting fast advances in accurate multianalyte monitoring in ISF toward in vivo measurements in animals and humans.

\section{EXPERIMENTAL SECTION}

Preparation of the MN Patch for the Potentiometric Determination of $\mathrm{pH}$. The $\mathrm{MN}$ patch for $\mathrm{pH}$ sensing consists of two solid MNs acting as working (WE) and reference (RE) electrodes, that are fixed in a silicon rubber substrate. This configuration is particularly suitable to access to the wearable technology owing to its flexibility and biocompatibility. The manufacturing process is illustrated in Figure 2a. More details on substrate fabrication and MN fixation are provided in the Supporting Information. The WE is a potentiometric $\mathrm{pH}$ electrode based on a three-layer structure of carbon ink, functionalized multiwalled carbon nanotubes (f-MWCNTs) as an ion-to-electron transducer and a hydrogen-selective membrane (HSM) (see Figure $2 \mathrm{~b}$ ). The $\mathrm{RE}$ consists of $\mathrm{a} \mathrm{Ag} / \mathrm{AgCl}$ layer covered by a poly(vinyl butyral) reference membrane (RM) cocktail (see the Supporting Information), which provides a high and constant chloride concentration in the solid-state RE, as previously demonstrated elsewhere. $^{29,30}$ The layer-by-layer structure of the RE is displayed in Figure $2 c$, whereas optical images of WE and RE are shown in Figure $2 \mathrm{~d}, \mathrm{e}$, respectively. Transdermal $\mathrm{pH}$ measurements in euthanized rats were performed by coupling the developed $\mathrm{MN}$ patch with a portable potentiometer (Figure $2 \mathrm{f}$ ). Note that all the rat-based experiments were carried out at the Karolinska University Hospital (Stockholm, Sweden) and assisted by the Operation Manager and Karolinska Experimental Research and Imaging Centre (KERIC) personnel. The rats were donated by KERIC and consisted of euthanized specimens that were previously used for other research purposes at KERIC. Importantly, the animals were not specifically euthanized for the purposes of our investigations but used as donated.

\section{RESULTS AND DISCUSSION}

The fabrication process for the $\mathrm{pH}$ WE was adapted from a recipe previously reported by our group. ${ }^{26}$ Thus, the deposition of the HSM was optimized considering two different approaches to incorporate the membrane cocktail: dip-coating and dropcasting. For both approaches, different membrane thicknesses were evaluated, which were obtained by changing the number of layers in dip-coating $(3,5,10,15$, and 300$)$ and both the number 
of layers $(1-3)$ and volume per layer $(0.5-3 \mu \mathrm{L})$ in dropcasting.

Figure 3a,b depicts the calibration graphs obtained by separate buffer solutions and using electrodes prepared by each different deposition protocol. As observed in Figure 3a, none of the proposed configurations fabricated via dip-coating provided a Nernstian response, with the highest slope $(34.3 \mathrm{mV})$ obtained using 15 layers of the membrane cocktail. Conversely, drop-casting deposition provided better results for both the slope and the linear range of response (LRR) (see Figure 3b). A Nernstian response was found for the configuration prepared by drop-casting three layers of $1 \mu \mathrm{L}$ of the membrane cocktail. This MN sensor presented $54.4 \mathrm{mV} \mathrm{dec}^{-1}$ and 8.5-5.0 as slope and LRR, respectively. The differences between both methods are likely explained by a better control of the layers' homogeneity by drop-casting rather than dip-coating, as suggested by images obtained using optical microscopy (see Figure S1 in the Supporting Information).

The optimized WE was combined with the MN RE to conform the final potentiometric MN-based patch. Notably, the performance of the MN RE was exhaustively characterized elsewhere, even proving its mechanical resilience during transdermal insertions. ${ }^{26}$ Figure $3 c$ compares the calibration graph $(n=3$, three successive measurements using the same electrode) for the $\mathrm{pH} \mathrm{MN}$ sensor against both a commercial Ag/ $\mathrm{AgCl} \mathrm{RE}$ (blue line) and the MN RE (black line). A very similar average slope was found in both cases: $54.6 \pm 0.6 \mathrm{mV} \mathrm{dec}^{-1}$ for the MN RE and $53.8 \pm 0.9 \mathrm{mV} \mathrm{dec}^{-1}$ for the commercial RE. An offset of $100 \mathrm{mV}$ between both sensors was observed, which is evidently associated to the change of the reference electrode as reported elsewhere. ${ }^{29}$ As a result, the MN RE was used in all further measurements.

First, the analytical performance of the developed MN patch was characterized using an in vitro approach in buffer media. Between-electrode reproducibility was evaluated by carrying out one calibration using three different $\mathrm{MN}$ patches, obtaining a \% $\mathrm{RSD}$ of 0.4 for the slope. Response repeatability, calculated from three consecutive calibration graphs performed with the same sensor, showed a \% RSD of 2.5 and 4.1 for the slope and intercept, respectively. Reversibility was evaluated recording 10 successive calibration curves with increasing and decreasing $\mathrm{pH}$ values alternatively (Figure $3 \mathrm{~d}$ ). The sensor displayed a variation for the slope and the intercept of 3.5 and $2.4 \%$, respectively, which reflect a rather good reversibility considering the entire experiment time scale (almost $2 \mathrm{~h}$ ) and the rather large $\mathrm{pH}$ changes in the experiment. Fast response time $\left(t_{95}<5 \mathrm{~s}\right)$ and very low long-term drift $\left(1.2 \pm 2.1 \mathrm{mV} \mathrm{h}^{-1}\right.$, in $10 \mathrm{mM} \mathrm{HCl}$ for a $16 \mathrm{~h}$ experiment, $n=3$ sensors, see Figure S2a in the Supporting Information) were also achieved. Overall, in view of all these results, the $\mathrm{pH} \mathrm{MN}$ patch presented excellent analytical features.

Next, the suitability of the MN patch for ISF analysis was evaluated through a selectivity study considering the major interferences found in this biological fluid, namely, $\mathrm{Na}^{+}, \mathrm{K}^{+}$, $\mathrm{Mg}^{2+}, \mathrm{Ca}^{2+}$, glucose, and urea. Table S1 collects the logarithmic selectivity coefficients $(n=3)$, calculated by the separate solution method. ${ }^{31}$ For all the interferences tested, selectivity coefficients were lower than the minimum value required for accurate measurements in ISF, which was estimated considering the highest concentration of each interference traditionally expected in ISF. ${ }^{32}$ Furthermore, the lack of synergistic effects between interferences was demonstrated by recording several calibration curves in AISF solution. Thus, Figure 3e presents the calibration graphs $(n=3)$ obtained in either buffer media (black line) or AISF (blue line). Similar slopes were observed in both media $\left(54.2 \pm 2.9 \mathrm{mV} \mathrm{dec}^{-1}\right.$ in AISF and $54.6 \pm 0.6 \mathrm{mV} \mathrm{dec}^{-1}$ in buffer) with the same LRR (from 8.5 to 5.0 ), which is indeed wide enough to cover expected $\mathrm{pH}$ values in ISF. Normal $\mathrm{pH}$ values in ISF range within 7.35 and 7.45 but may be altered due to the influence of diseases or health disorders/infections. For example, in the case of cancer, ISF is known to vary from 6.2 to 6.9. ${ }^{33}$ In addition, the long-term drift in AISF was rather acceptable $\left(0.6 \mathrm{mV} \mathrm{h}^{-1}\right.$ for $2 \mathrm{~h}$ experiment), as shown in Figure $\mathrm{S} 2 \mathrm{~b}$ in the Supporting Information.

Subsequently, ex vivo evaluation of the $\mathrm{MN}$ patch for transdermal $\mathrm{pH}$ detection in the ISF of different types of animal skins was accomplished to investigate two key aspects: (i) the response of the developed MN sensors is not altered and/or the external modification detached from the $\mathrm{MN}$ with the skin insertion; and (ii) appropriate accuracy of the measurements by means of a carefully designed validation protocol. One approach widely proposed in the literature to assess $\mathrm{MN}$ sensor resistance to skin penetration consists of using agarose hydrogels in contact with AISF to mimic the real skin system. ${ }^{34,35}$ The resiliency of MN sensors is evaluated by comparing the calibration parameters before and after the insertion into the hydrogel. Then, the suitability to use an external calibration for transdermal quantification of the analyte is assessed by measuring different concentrations in AISF that reach the hydrogel by diffusion. However, this approach does not provide a realistic evaluation of the ability of $\mathrm{MN}$ sensors to perform effective skin insertion because the hydrogel is softer and easier to penetrate than any real skin. In contrast, a closer approach to in vivo analysis utilizes animal skin instead. ${ }^{26,36,37}$ This methodology allows for a better evaluation of viscoelasticity and the adherence of undesired substances to the MN (including biofouling), thus providing close conditions to the further on-body use in animals and/or humans.

In this work, sensor resilience to skin penetration was assessed by manually inserting the $\mathrm{MN}$ patch in pieces of chicken, porcine, and rat skin several times (1, 3, 5, and 10 times) and registering the calibration curve in AISF afterward. This was compared with an initial calibration recorded prior to any insertion. Figure $4 \mathrm{a}$ shows the results obtained in chicken skin, which is the thinnest skin and the easiest to penetrate out of the three tested types of skin. No significant changes were observed in the slope after ten insertions (the initial slope was $52.0 \mathrm{mV}$

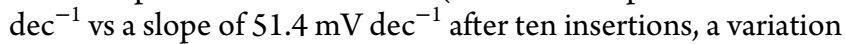
coefficient of $1.8 \%$ ). Furthermore, if all the calibrations are considered, the obtained \% RSD for the slope and intercept was 2.1 and 1.8, respectively. Thus, it is concluded that the response of the MN patch is not altered during insertion in chicken skin. Furthermore, the found RSD lies within the variation observed in the repeatability studies of the electrodes in the in vitro characterization (2.5 and 4.1 for the slope and intercept $(n=3)$ respectively). Accordingly, variations found in the standard electrode potential of the MNs with consecutive skin insertions can be likely attributed to an inherit variation of the sensor response rather than a perturbation due to the insertion cycles.

Analogous results were obtained in porcine skin (Figure $4 b$ ), with the initial slope of $51.3 \mathrm{mV} \mathrm{dec}^{-1}$ versus a slope of $52.7 \mathrm{mV}$ $\mathrm{dec}^{-1}$ after ten insertions, \% RSD of 1.5 and 1.2 for the slope and intercept considering all the calibration graphs. Significantly, the appropriate results found in porcine skin are particularly promising for future application of MN sensors in humans because this has striking similarities to the human skin in terms of general structure, thickness, hair follicle content, pigmenta- 

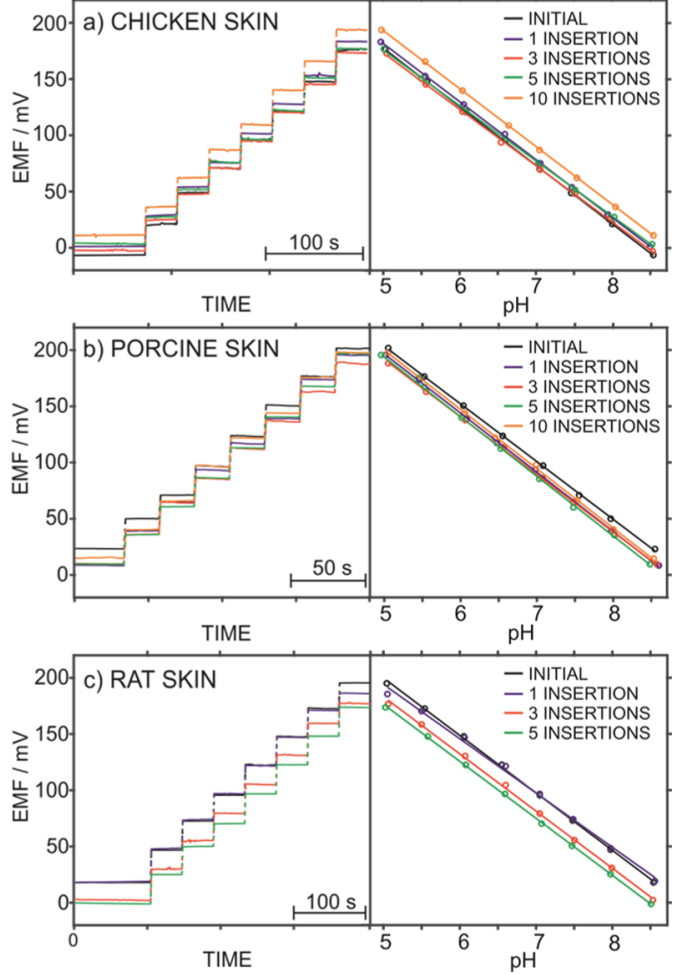

Figure 4. Dynamic responses and the corresponding calibration graphs observed for $\mathrm{pH}$ before and after several insertions into (a) chicken, (b) porcine, and (c) rat skin.

tion, collagen, and lipid composition. ${ }^{38}$ Finally, Figure $4 \mathrm{c}$ displays the results obtained in rat skin. Similar calibration curves were also obtained after up to five insertions: with the initial slope of $51.1 \mathrm{mV} \mathrm{dec}{ }^{-1}$ versus a slope of $50.7 \mathrm{mV} \mathrm{dec}^{-1}$ after five insertions and \% RSD of 2.3 and 2.8 for the slope and intercept considering all the calibration graphs.

When using rat skin in the ex vivo studies (Figure 4c), the intercept was found to slightly shift to less positive values after each insertion. Although the variation after 5 insertions was acceptable, higher number of insertions would require a recalibration of the sensor to correct this drift. Notably, onbody experiments in rats herein presented are based in three skin insertions and the electrodes were calibrated before and after the transdermal measurements to minimize any error in the $\mathrm{pH}$ quantification arising from a change in the calibration graph.

The absence of any attachment of biological material in the MNs due to skin insertion and/or contact with the tissues was confirmed by microscopic images: a comparison of the images taken before (Figure S1b) and after (Figure S1c) penetration in chicken skin is presented in the Supporting Information. As observed, no particles or pieces are attached to the $\mathrm{MN}$ sensor after the insertion. In addition, no sign about the deterioration and/or detachment of the sensing element was detected. This confirms, in turn, the absence of marked variations in the electrode calibration after several insertions (Figure 4a), hence pointing out the absence of biofouling effects.

The second part of the ex vivo assays focused on the validation of transdermal $\mathrm{pH}$ measurements with the developed $\mathrm{MN}$ patch once inserted in animal skin and therefore demonstrating the suitability of an external calibration of the sensor for the $\mathrm{pH}$ quantification in further on-body studies. We designed these tests on the basis of skin pieces that were conditioned $24 \mathrm{~h}$ at different $\mathrm{pH}$. This experiment is not trivial, and one should not assume that the intradermal concentration of any analyte can be easily modified just by direct contact of the skin with an external solution of the desired analyte concentration. For example, Senel et al. reported a lower chronoamperometric response for urea when MN sensors were measuring inside phantom gel than that expected according to the concentration fixed in external AISF. ${ }^{39}$ This behavior was attributed to the slow diffusion of urea in phantom gel. Similar conclusions were drawn in our previous studies regarding the determination of $\mathrm{K}^{+}$in chicken skin using potentiometric MN sensors. ${ }^{26}$ It was demonstrated that the lower potassium concentration determined inside the skin as compared to the concentration in the AISF solution that soaked the skin was due to the diffusion and distribution of the ion driven by the concentration gradient at the skin-AISF interface. $^{26}$

In this paper, we opted for performing experiments by conditioning the pieces of animal skin with a known analyte concentration for a $24 \mathrm{~h}$ process and then measuring the transdermal $\mathrm{pH}$ with the MN patch (Figure 5a). Alternatively, the concentration inside the skin can be measured by mincing and digestion followed by analysis with reference analytical techniques. ${ }^{37}$ However, this option is not suitable for the particular case of $\mathrm{pH}$ determination because acid digestion will affect the measurement. To confirm that $24 \mathrm{~h}$ conditioning is enough to reach the desired $\mathrm{pH}$ inside the skin, we collected the ISF by means of a custom-made system based on hollow MNs and measured the $\mathrm{pH}$ with a commercial micro-pH electrode. The collection device was based on a commercially available hub with hollow MNs connected to a peristaltic pump (see the Supporting Information for more details). We found that the $\mathrm{pH}$ in the ISF was very similar to that fixed in the conditioning solution (see Table S2 in the Supporting Information). In addition, the $\mathrm{pH}$ of the conditioning solution was measured by means of a commercial $\mathrm{pH}$ meter to ensure that $\mathrm{pH}$ was retained during $24 \mathrm{~h}$.
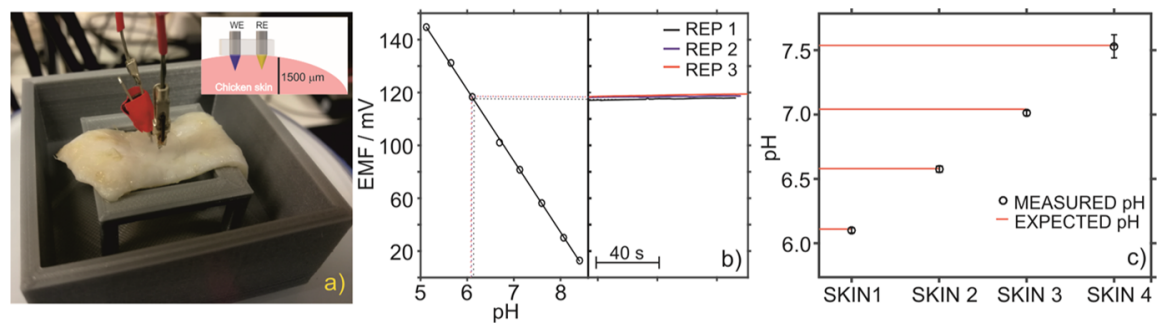

Figure 5. (a) Setup for ex vivo measurements performed with the $\mathrm{MN}$ patch inserted in chicken skin conditioned at different $\mathrm{pH}$ values. Inset: scheme of the MN insertion. (b) External calibration graph and three consecutive potentiometric responses obtained during the measurement of chicken skin at $\mathrm{pH}$ 6.0. (c) Measured and expected values in ex vivo $\mathrm{pH}$ measurements of four chicken skin pieces conditioned at different $\mathrm{pH}$ values. 

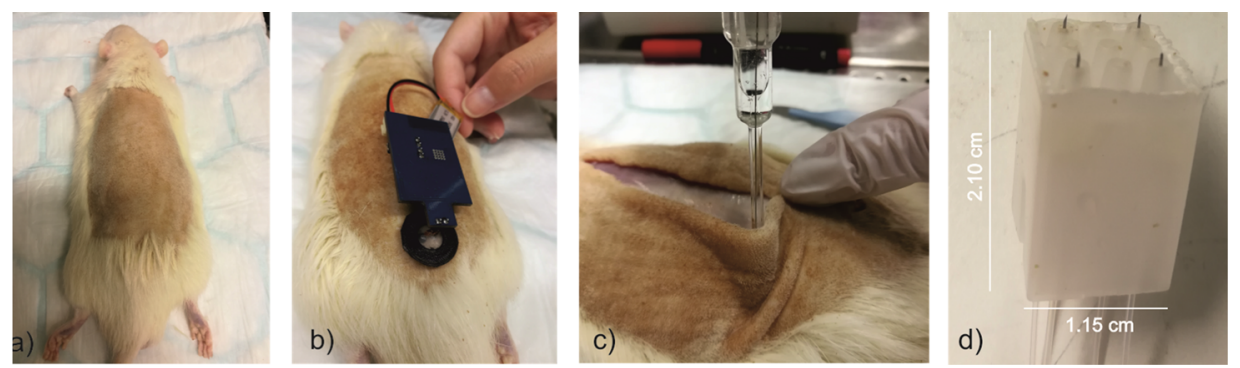

Figure 6. Pictures of on-body measurements in a euthanized rat specimen: (a) shaved rat prepared for the insertion of the $\mathrm{MN}$ patch. (b) $\mathrm{pH} \mathrm{MN}$ patch coupled with the potentiometric electronic board and providing transdermal measurements in the rat back. (c) Subcutaneous measurement of $\mathrm{pH}$ using a micro-pH meter. (d) Home-made tool for ISF collection based on a hollow MN hub connected to a peristaltic pump.

Transdermal potentiometric responses inside each type of skin were recorded by triplicate and $\mathrm{pH}$ values were calculated by extrapolating average potentials into an external calibration performed in AISF (see Figure 5b). Figure 5c displays the results obtained for four chicken skin pieces conditioned at different $\mathrm{pH}$ values, with black circles representing the values measured by the $\mathrm{MN}$ patch and red lines indicating the expected $\mathrm{pH}$ values. The accuracy and precision of the transdermal measurements using the $\mathrm{MN}$ results in the ex vivo experiments are the perfect preamble for further animal-based tests based on the on-body use of the new MN patch.

Prior to any in vivo tests in rats and humans, it is mandatory to demonstrate the biocompatibility level of the $\mathrm{MN}$ patch ensuring that no toxic effect is caused by direct contact, leaching, or detachment of the sensing element. In this context, we have recently reported on the cytotoxicity of all the materials used in the $\mathrm{pH}$ MN sensor herein developed. ${ }^{26,28}$ Briefly, cell viability and cell proliferation studies using fibroblasts were carried out with bare MNs, MNs coated with either carbon or $\mathrm{Ag} / \mathrm{AgCl}$ ink, f-MWCNT, and the HSM. In all cases, after $96 \mathrm{~h}$ of incubation, the total number of counted cells was comparable to control conditions (fibroblasts immersed in culture media and without the presence of material/compound), indicating the absence of cytotoxicity effects. However, this outcome should not be generalized to any MN-based ISE because, unlike HSM, other membrane compounds, such valinomycin-based membranes for potassium or nonactin membranes for ammonium, revealed a certain level of cytotoxicity after $96 \mathrm{~h}^{28}$

Then, in vivo tests should come accompanied by validated measurements using a gold standard technique, although the literature is really scarce in this issue. Indeed, the implementation of this validation is not trivial due to the difficulty of extracting enough volume of the same ISF that is measured by the MN. We explored two different validation protocols for the $\mathrm{pH} \mathrm{MN}$ tests in rats. The first approach measures subcutaneous $\mathrm{pH}$ by means of a commercial $\mathrm{pH}$ microelectrode after opening the specimen. The second protocol is based on ISF extraction using a home-made system based on a commercially available hollow MN hub (see the Supporting Information for more details). Interstitial $\mathrm{pH}$ was measured in seven rats with different genders and ages (see Table S3 in the Supporting Information) using the newly developed $\mathrm{MN}$ patch and the two validation protocols. All measurements were performed sequentially in one rat before moving to the next one to minimize $\mathrm{pH}$ changes due to evaporation and/or stop in blood flow. Notably, the rats were donated by KERIC and consisted of euthanized specimens that were previously used for other research purposes at KERIC.

To implant the MN sensors, the back of the rats was shaved to facilitate $\mathrm{MN}$ insertion and visual inspection (Figure 6a).
Subsequently, the MN patch was inserted in the rat back and secured with a ring of polyurethane (PU) to prevent any movement of the MNs during the on-body measurements. Finally, the patch was connected to a wireless potentiometric board and the response was recorded until the steady-state potential was reached, $40 \mathrm{~s}$ approx. (see Figure 6b). Three consecutive insertions were performed to obtain the measurement in triplicate and to confirm that the sensing element is not detached from the MN.

A total of 7 rats were tested, with the rat number 5 monitored with two different (and twin) MN patches (Table S3). In none of the cases, we could visually detect any modification of the MN after transdermal measurements. No significant alteration was detected in the potentiometric response upon increasing insertions either. Moreover, we found variation coefficients of less than $1 \%$ in all the tested rats, except for the first measurement round in rat number 5 and in rat number 6 , where ca. $5 \%$ of variation was observed. This higher variation in triplicate measurements specifically obtained in two specimens is likely associated to the quality of the hand-made fabricated MN patches: the three measurements accomplished in rat 5 provided values (in this order) of 7.51, 7.03, and 6.60. Then, when measurements were repeated in the same rat but with a different MN patch, the provided values were $6.36,6.48$, and 6.48 , with a variation coefficient in the range of the $1 \%$ as in the majority of the rats. Thus, in the case of the first measurement round, the real $\mathrm{pH}$ is seemingly within the two last values, with the very first measurement interpreted as an outlier.

After the transdermal measurements, the first validation method was carried out by means of an incision in the rat's back, exposing the subcutaneous ISF and measuring the $\mathrm{pH}$ with a commercial microelectrode (Figure 6c). For the second validation method, ISF was directly extracted from the rat back with the MN hub (Figure 6d, see the Supporting Information for more details). ISF extraction was prolonged for $30 \mathrm{~min}$, obtaining significantly different sample volumes for each rat, ranging from nonmeasurable volume (almost zero) to more than $100 \mu \mathrm{L}$ (Table S3 in the Supporting Information). The $\mathrm{pH}$ of the collected ISF was measured with an ultramicro $\mathrm{pH}$ electrode, specifically designed for low-volume sample analysis $(\sim 0.5 \mu \mathrm{L})$. Only for one rat, it was not possible to extract enough ISF to be measured with the $\mathrm{pH}$ meter. Interestingly, the highest volumes of ISF were extracted from the youngest rats ( 2 months), which is likely attributed to both smoother skin and higher hydration level. This combination enhances hydraulic conductivity of tissue, leading to spacing tissue fibers that facilitate ISF extraction. ${ }^{40}$

Individual correlations between the three types of measurements were statistically evaluated to quantify the accuracy of the 
pH MN patch: separate paired sample $t$-tests (also known as the dependent sample $t$-test) were carried out. Notably, since measurements performed on the same rat are treated as a single pair of observation, differences in $\mathrm{pH}$ among different rats will not influence the test. Graphical representations of the paired sample $t$-test are displayed in Figure 7, with Figure 7a presenting
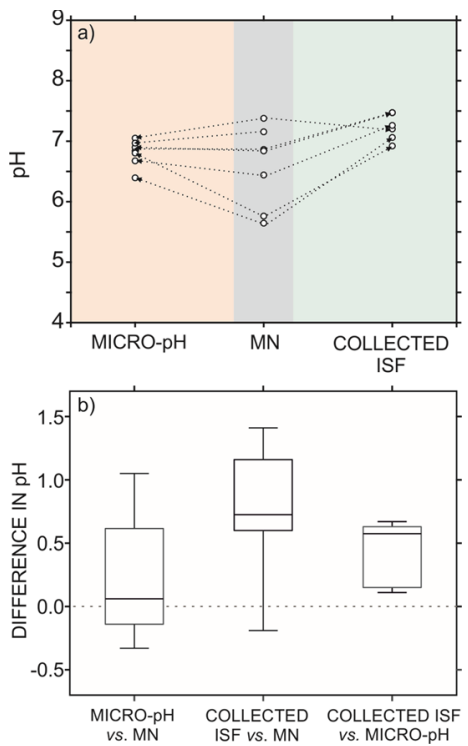

Figure 7. (a) Comparison of $\mathrm{pH}$ measurements with the $\mathrm{MN}$ patch and two different validation procedures: subcutaneous $\mathrm{pH}$ measurements with the micro-pH meter and ISF extraction. Connected lines represent values obtained for the same rat. (b) Paired sample $t$-test box plot representing statistically analyzed differences in $\mathrm{pH}$ measurements with the MN patch, subcutaneous data, and ISF extraction. Gray dotted line represents the null hypothesis.

the direction in the variation of the $\mathrm{pH}$ measurements in each single rat (comparing the MN with each validation strategy), and Figure $7 \mathrm{~b}$ being a box and whisker plot of the measured differences between the three methods. Attending first to the comparison of $\mathrm{MN}$ results with subcutaneous measurements and considering a $95 \%$ of confidence level, the $t_{\text {calc }}=1.2$ was lower than $t_{\text {stat }}=2.5$ and hence there were no statistically meaningful differences between both methods (accepting the null hypothesis, see Figure $7 \mathrm{~b}$ ). This result is easily visualized in Figure $7 \mathrm{a}$, where no clear direction in variation appears. Contrarily, the paired sample $t$-test indicated that there was a statistically significant difference between $\mathrm{MN}$ results and the $\mathrm{pH}$ measurements in the collected ISF $\left(t_{\text {calc }}=3.27>t_{\text {stat }}=2.57\right)$.

A further comparison between the $\mathrm{pH}$ provided by MNs and the subcutaneous measurements can be accomplished by directly comparing the differences in values (see Table S3). In all the cases, this difference is lower than $0.3 \mathrm{pH}$ units, except for rat number 4 and number 7 (ca. $1 \mathrm{pH}$ unit). Despite the results being within the bounds of experimental variance, ISF evaporation is hard to be controlled in subcutaneous measurements when the skin is exposed to the air, and this may result in significant variations compared to the MN data. Transdermal measurements are indeed advantageous compared with the two validation techniques, which is required for sample and/or specimen manipulation. On the other hand, as observed in Figure $7 \mathrm{a}$, ISF extraction tends to provide $\mathrm{pH}$ values higher than the MN patch. Thus, the two validation methods are not providing the same information and evidently, at least one of them presents some systematic error. Moreover, comparison of the two validation methods results in $t_{\text {calc }}=4.4>t_{\text {stat }}=2.6$, meaning that the results are statistically different (see Figure $7 \mathrm{~b}$ ). A plausible explanation of this result is that ISF extraction takes place for a long time period $(30 \mathrm{~min})$ in which blood flow has been stopped because the rat is no longer alive, therefore affecting normal diffusion processes between blood and ISF.

The same observation was reported by Mani et al. for anaesthetized mice: $\mathrm{pH}$ values measured with $\mathrm{MN}$ sensors in CSF and bladder were slightly lower than those measured using commercial $\mathrm{pH}$ electrodes after fluid extraction. ${ }^{16}$ Authors attributed the observed differences to a change in the sample $\mathrm{pH}$ caused by the removal of biological fluids from their buffered (physiological) conditions. Also, subcutaneous measurements are directly accomplished in the rat, and, therefore, less marked alterations in the sample are expected. As a result, subcutaneous measurements seem to be more reliable as the validation procedure. Nevertheless, it should be noted that this method requires surgery, and therefore, future efforts should be devoted to the development of noninvasive and reliable validation protocols toward an adequate validation of pure in vivo measurements in animals and humans.

\section{CONCLUSIONS}

A fully validated potentiometric $\mathrm{pH} \mathrm{MN}$ sensor for transdermal $\mathrm{pH}$ measurements in ISF has been presented. This is the first time that such a sensor is demonstrated for on-body measurements in rats after a deep in vitro and ex vivo characterization at the laboratory scale. Stainless-steel solid MNs were modified following a layer-by-layer approach to provide robust and reliable $\mathrm{pH}$ measurements. Analytical characterization of the MN patch under in vitro conditions demonstrated suitable Nernstian response, excellent repeatability and reproducibility, fast response time, adequate drift, and an LRR wide enough to cover both physiological and abnormal $\mathrm{pH}$ levels in ISF. Ex vivo assays using chicken, porcine, and rat skin revealed that the new $\mathrm{MN}$ patch is resilient to skin insertion and provides high precision and accuracy, as inferred from an ex vivo validation using preconditioned pieces of animal skin. The MN patch was successfully implemented into on-body assays in rats, measuring interstitial $\mathrm{pH}$ in seven specimens. Two different validation protocols were evaluated, concluding that subcutaneous $\mathrm{pH}$ measurements are more reliable than the analysis of extracted ISF samples. Overall, this work provides a clear guide on how to properly characterize and validate the analytical performance of newly developed MN sensors for any type of analyte (ions and biomolecules), starting from in vitro conditions and reaching onbody measurements. This is indeed a key aspect in making meaningful advances in the development and application of wearable eHealth devices based on minimally invasive MNs.

\section{ASSOCIATED CONTENT}

\section{SI Supporting Information}

The Supporting Information is available free of charge at https://pubs.acs.org/doi/10.1021/acssensors.0c02397.

Experimental details, logarithmic selectivity coefficients for the $\mathrm{pH}$ MN electrode; $\mathrm{pH}$ monitoring of chicken skin conditioned for $24 \mathrm{~h}$; results obtained in rat assays via MNs, micro $\mathrm{pH}$ meter, ISF collection; and optical microscopic images of $\mathrm{pH} \mathrm{MN}$ sensors (PDF) 


\section{AUTHOR INFORMATION}

\section{Corresponding Author}

Gastón A. Crespo - Department of Chemistry, School of Engineering Science in Chemistry, Biochemistry and Health, Royal Institute of Technology, KTH, SE-100 44 Stockholm, Sweden; ๑ orcid.org/0000-0002-1221-3906; Email: gapc@ kth.se

\section{Authors}

Juan José García-Guzmán - Department of Chemistry, School of Engineering Science in Chemistry, Biochemistry and Health, Royal Institute of Technology, KTH, SE-100 44 Stockholm, Sweden

Clara Pérez-Ràfols - Department of Chemistry, School of Engineering Science in Chemistry, Biochemistry and Health, Royal Institute of Technology, KTH, SE-100 44 Stockholm, Sweden

María Cuartero - Department of Chemistry, School of Engineering Science in Chemistry, Biochemistry and Health, Royal Institute of Technology, KTH, SE-100 44 Stockholm, Sweden

Complete contact information is available at:

https://pubs.acs.org/10.1021/acssensors.0c02397

\section{Notes}

The authors declare no competing financial interest.

\section{ACKNOWLEDGMENTS}

We acknowledge the financial support from the Swedish Research Council (Project Grant VR-2017-4887), Stiftelsen Olle Engkvist Byggmästare (Project Grant 204-0214), and the Novo Nordisk Fonden (Exploratory Pre-Seed Grant 19OC0056171). We also acknowledge the facilities and support provided by Karolinska Experimental Research and Imaging Centre (KERIC) for the rat-based assays. We thank Emil Ekelund for his support on the development of the wireless potentiometric board used for transdermal $\mathrm{pH}$ monitoring in rats.

\section{REFERENCES}

(1) Liu, G.-S.; Kong, Y.; Wang, Y.; Luo, Y.; Fan, X.; Xie, X.; Yang, B.R.; Wu, M. X. Microneedles for transdermal diagnostics: Recent advances and new horizons. Biomaterials 2020, 232, 119740.

(2) García-Guzmán, J.J.; Pérez-Ràfols, C.; Cuartero, M.; Crespo, G. A. Microneedle Based Electrochemical (Bio)Sensing: Towards Decentralized and Continuous Health Status Monitoring. Trends Anal. Chem. 2021, 135, 116148.

(3) Yang, Y.; Gao, W. Wearable and flexible electronics for continuous molecular monitoring. Chem. Soc. Rev. 2019, 48, 1465-1491.

(4) Wiorek, A.; Parrilla, M.; Cuartero, M.; Crespo, G. A. Epidermal Patch with Glucose Biosensor: $\mathrm{pH}$ and Temperature Correction toward More Accurate Sweat Analysis during Sport Practice. Anal. Chem. 2020, 92, 10153-10161.

(5) Parrilla, M.; Cuartero, M.; Crespo, G. A. Wearable potentiometric ion sensors. Trends Anal. Chem. 2019, 110, 303-320.

(6) Cuartero, M.; Parrilla, M.; Crespo, G. Wearable potentiometric sensors for medical applications. Sensors 2019, 19, 363.

(7) Niedzwiecki, M. M.; Samant, P.; Walker, D. I.; Tran, V.; Jones, D. P.; Prausnitz, M. R.; Miller, G. W. Human suction blister fluid composition determined using high-resolution metabolomics. Anal. Chem. 2018, 90, 3786-3792.

(8) Tran, B. Q.; Miller, P. R.; Taylor, R. M.; Boyd, G.; Mach, P. M.; Rosenzweig, C. N.; Baca, J. T.; Polsky, R.; Glaros, T. Proteomic characterization of dermal interstitial fluid extracted using a novel microneedle-assisted technique. J. Proteome Res. 2018, 17, 479-485.
(9) Miller, P. R.; Taylor, R. M.; Tran, B. Q.; Boyd, G.; Glaros, T.; Chavez, V. H.; Krishnakumar, R.; Sinha, A.; Poorey, K.; Williams, K. P.; Branda, S. S.; Baca, J. T.; Polsky, R. Extraction and biomolecular analysis of dermal interstitial fluid collected with hollow microneedles. Commun. Biol. 2018, 1, 173.

(10) Wang, P. M.; Cornwell, M.; Prausnitz, M. R. Minimally invasive extraction of dermal interstitial fluid for glucose monitoring using microneedles. Diabetes Technol. Ther. 2005, 7, 131-141.

(11) Mukerjee, E. V.; Collins, S. D.; Isseroff, R. R.; Smith, R. L. Microneedle array for transdermal biological fluid extraction and in situ analysis. Sens. Actuators, A 2004, 114, 267-275.

(12) Kim, K. B.; Choi, H.; Jung, H. J.; Oh, Y.-J.; Cho, C.-H.; Min, J. H.; Yoon, S.; Kim, J.; Cho, S. J.; Cha, H. J. Mussel-inspired enzyme immobilization and dual real-time compensation algorithms for durable and accurate continuous glucose monitoring. Biosens. Bioelectron. 2019, 143, 111622.

(13) Ribet, F.; Stemme, G.; Roxhed, N. Real-time intradermal continuous glucose monitoring using a minimally invasive microneedlebased system. Biomed. Microdevices 2018, 20, 101.

(14) Jina, A.; Tierney, M. J.; Tamada, J. A.; McGill, S.; Desai, S.; Chua, B.; Chang, A.; Christiansen, M. Design, development, and evaluation of a novel microneedle array-based continuous glucose monitor. J. Diabetes Sci. Technol. 2014, 8, 483-487.

(15) Chua, B.; Desai, S. P.; Tierney, M. J.; Tamada, J. A.; Jina, A. N. Effect of microneedles shape on skin penetration and minimally invasive continuous glucose monitoring in vivo. Sens. Actuators, A 2013, 203, 373-381.

(16) Mani, G. K.; Miyakoda, K.; Saito, A.; Yasoda, Y.; Kajiwara, K.; Kimura, M.; Tsuchiya, K. Microneedle $\mathrm{pH}$ sensor: direct, label-free, real-time detection of cerebrospinal fluid and bladder $\mathrm{pH}$. ACS Appl. Mater. Interfaces 2017, 9, 21651-21659.

(17) Hegarty, C.; McConville, A.; McGlynn, R. J.; Mariotti, D.; Davis, $\mathrm{J}$. Design of composite microneedle sensor systems for the measurement of transdermal pH. Mater. Chem. Phys. 2019, 227, 340-346.

(18) Park, J. E.; Yonet-Tanyeri, N.; Vander Ende, E.; Henry, A.-I.; Perez White, B. E.; Mrksich, M.; Van Duyne, R. P. Plasmonic Microneedle Arrays for in Situ Sensing with Surface-Enhanced Raman Spectroscopy (SERS). Nano Lett. 2019, 19, 6862-6868.

(19) Zuliani, C.; Ng, F. S.; Alenda, A.; Eftekhar, A.; Peters, N. S.; Toumazou, C. An array of individually addressable micro-needles for mapping pH distributions. Analyst 2016, 141, 4659-4666.

(20) Zhou, J.-X.; Ding, F.; Tang, L.-N.; Li, T.; Li, Y.-H.; Zhang, Y.-J.; Gong, H.-Y.; Li, Y.-T.; Zhang, G.-J. Monitoring of $\mathrm{pH}$ changes in a live rat brain with MoS2/PAN functionalized microneedles. Analyst 2018, 143, 4469-4475.

(21) Witten, M. L.; Sheppard, P. R.; Witten, B. L. Tungsten toxicity. Chem.-Biol. Interact. 2012, 196, 87-88.

(22) Molybdenum Disulfide $\left(\mathrm{MoS}_{2}\right)$ Powder Safety Data Sheet; US Research Nanomaterials, Inc., 2016. www.us-nano.com.

(23) Iridium Oxide Safety Data Sheet; Alfa Aesar, 2020. www.alfa.com. (24) Manjakkal, L.; Dervin, S.; Dahiya, R. Flexible potentiometric pH sensors for wearable systems. RSC Adv. 2020, 10, 8594-8617.

(25) Manjakkal, L.; Szwagierczak, D.; Dahiya, R. Metal oxides based electrochemical $\mathrm{pH}$ sensors: Current progress and future perspectives. Prog. Mater. Sci. 2020, 109, 100635.

(26) Parrilla, M.; Cuartero, M.; Padrell Sánchez, S.; Rajabi, M.; Roxhed, N.; Niklaus, F.; Crespo, G. A. Wearable All-Solid-State Potentiometric Microneedle Patch for Intradermal Potassium Detection. Anal. Chem. 2019, 91, 1578-1586.

(27) Hu, J.; Stein, A.; Bühlmann, P. Rational design of all-solid-state ion-selective electrodes and reference electrodes. Trends Anal. Chem. 2016, 76, 102-114.

(28) Cánovas, R.; Padrell Sánchez, S.; Parrilla, M.; Cuartero, M.; Crespo, G. A. Cytotoxicity study of ionophore-based membranes: toward on-body and in vivo ion sensing. ACS Sens. 2019, 4, 2524-2535.

(29) Guinovart, T.; Crespo, G. A.; Rius, F. X.; Andrade, F. J. A reference electrode based on polyvinyl butyral (PVB) polymer for decentralized chemical measurements. Anal. Chim. Acta 2014, 821, 7280 . 
(30) Cuartero, M.; del Río, J. S.; Ortuño, J. A.; Rius, F. X.; Andrade, F. J. Rubber-based substrates modified with carbon nanotubes inks to build flexible electrochemical sensors. Anal. Chim. Acta 2014, 827, 95102.

(31) Bakker, E.; Bühlmann, P. Selectivity of Potentiometric Ion Sensors. Anal. Chem. 2000, 72, 1127-1133.

(32) Bretag, A. H. Synthetic interstitial fluid for isolated mammalian tissue. Life Sci. 1969, 8, 319-329.

(33) Cardone, R. A.; Casavola, V.; Reshkin, S. J. The role of disturbed $\mathrm{pH}$ dynamics and the $\mathrm{Na}+/ \mathrm{H}+$ exchanger in metastasis. Nat. Rev. Cancer 2005, 5, 786-795.

(34) Bollella, P.; Sharma, S.; Cass, A. E. G.; Antiochia, R. Minimallyinvasive microneedle-based biosensor array for simultaneous lactate and glucose monitoring in artificial interstitial fluid. Electroanalysis 2019, 31, 374-382.

(35) Mishra, R. K.; Goud, K. Y.; Li, Z.; Moonla, C.; Mohamed, M. A.; Tehrani, F.; Teymourian, H.; Wang, J. Continuous opioid monitoring along with nerve agents on a wearable microneedle sensor array. J. Am. Chem. Soc. 2020, 142, 5991-5995.

(36) Mohan, A. M. V.; Windmiller, J. R.; Mishra, R. K.; Wang, J. Continuous minimally-invasive alcohol monitoring using microneedle sensor arrays. Biosens. Bioelectron. 2017, 91, 574-579.

(37) Jin, Q.; Chen, H.-J.; Li, X.; Huang, X.; Wu, Q.; He, G.; Hang, T.; Yang, C.; Jiang, Z.; Li, E.; Zhang, A.; Lin, Z.; Liu, F.; Xie, X. Reduced graphene oxide nanohybrid-assembled microneedles as mini-invasive electrodes for real-time transdermal biosensing. Small 2019, 15, 1804298.

(38) Summerfield, A.; Meurens, F.; Ricklin, M. E. The immunology of the porcine skin and its value as a model for human skin. Mol. Immunol. 2015, 66, 14-21.

(39) Senel, M.; Dervisevic, M.; Voelcker, N. H. Gold microneedles fabricated by casting of gold ink used for urea sensing. Mater. Lett. 2019, 243, 50-53.

(40) Kolluru, C.; Williams, M.; Chae, J.; Prausnitz, M. R. Recruitment and Collection of Dermal Interstitial Fluid Using a Microneedle Patch. Adv. Healthcare Mater. 2019, 8, 1801262. 